\title{
One-loop renormalization of the chiral Lagrangian for spinless matter fields in the $\mathrm{SU}(N)$ fundamental representation
}

\author{
Meng-Lin Du ${ }^{a, *}$, Feng-Kun Guo ${ }^{b, \dagger}$, Ulf-G. Meißner ${ }^{a, c, \ddagger}$ \\ ${ }^{a}$ Helmholtz-Institut für Strahlen- und Kernphysik and Bethe Center for Theoretical Physics, \\ Universität Bonn, D-53115 Bonn, Germany \\ ${ }^{b}$ CAS Key Laboratory of Theoretical Physics, Institute of Theoretical Physics, \\ Chinese Academy of Science, Beijing 100190, China \\ ${ }^{c}$ Institute for Advanced Simulation, Institut für Kernphysik and Jülich Center for Hadron Physics, \\ Forschungszentrum Jülich, D-52425 Jülich, Germany
}

September 11, 2018

\begin{abstract}
We perform the leading one-loop renormalization of the chiral Lagrangian for spinless matter fields living in the fundamental representation of $\mathrm{SU}(N)$. The Lagrangian can also be applied to any theory with a spontaneous symmetry breaking of $\mathrm{SU}(N)_{L} \times \mathrm{SU}(N)_{R}$ to $\mathrm{SU}(N)_{V}$ and spinless matter fields in the fundamental representation. For QCD, the matter fields can be kaons or pseudoscalar heavy mesons. Using the background field method and heat kernel expansion techniques, the divergences of the one-loop effective generating functional for correlation functions of single matter fields are calculated up to $\mathcal{O}\left(p^{3}\right)$. They are absorbed by counterterms not only from the third order but also from the second order chiral Lagrangian.
\end{abstract}

\footnotetext{
${ }^{*}$ E-mail address: du@hiskp.uni-bonn.de

${ }^{\dagger}$ Emerging leader. E-mail address: fkguo@itp.ac.cn

${ }^{\ddagger}$ E-mail address: meissner@hiskp.uni-bonn.de
} 


\section{Introduction}

Chiral perturbation theory (ChPT) provides a systematic and successful approach for investigating the low-energy behavior of the Goldstone bosons of the spontaneous symmetry breaking in QCD [1 3]. For quantum chromodynamics (QCD), the Lagrangian has a chiral symmetry $\mathrm{SU}(N)_{L} \times \mathrm{SU}(N)_{R}$ in the limit of vanishing quark masses, where $N$ is the number of flavors under consideration 1 , which is spontaneously broken to the vector subgroup $\mathrm{SU}(N)_{V}$. The Goldstone bosons are provided by the lowest-lying pseudoscalar mesons, to be denoted by $\phi$. Since the masses of the $u, d$ (and $s$ ) quarks are small, they can be treated as a perturbation, and the explicit chiral symmetry breaking due to the quark masses can be built into ChPT. In this spirit, the chiral Lagrangian can be constructed order by order in a double expansion in the momenta of Goldstone bosons, denoted by $p$, and the light quark masses with $m_{q}=\mathcal{O}\left(p^{2}\right)$ (at a fixed ratio of $p^{2} / m_{q}$ ). The corresponding coefficients are called lowenergy constants (LECs) which have both finite and divergent parts. To a specific order, both tree level and loop graphs up to that order are necessary. Under the proper power counting scheme [1, when one calculates a physical observable, the divergence arising from loops is cancelled by that of the LECs in the Lagrangian of the proper order, and one gets a finite and renormalization scale independent result.

Matter fields which are not the Goldstone bosons can be included in ChPT as well. However, because of the mass of the matter field does not vanish in the chiral limit, it presents a new energy scale which needs to be treated with caution. Because of the introduction of this new energy scale, the naive power counting is spoiled by the loops containing the matter field propagators [4]. Various methods were suggested to restore a consistent power counting. For the case of matter fields being baryons, there are methods such as the heavybaryon ChPT [5, 6] and manifestly Lorentz invariant infrared regularization [7, 9] as well as the extended-on-mass-shell scheme [10,11]. In the latter case, since the power counting breaking terms are polynomials of the external momenta and quark masses, they can be absorbed into the redefinition of the LECs. Generally, because of the new energy scale, contrary to the purely Goldstone boson ChPT, the ultraviolet (UV) divergences generated by the loops not only shift the higher order LECs, but also the lower order LECs (even the leading order ones in certain cases). The same situation happens in the extension of ChPT to include any other matter fields including ChPT for heavy-flavor hadrons [12 14] and SU(2) ChPT for $K \pi$ scattering [15].

In this paper, we will study the one-loop renormalization of the chiral Lagrangian for spinless (scalar or peudoscalar) matter fields in the fundamental representation of $\mathrm{SU}(N)$. This kind of theory can be applied to the scattering between light pseudoscalar mesons and pseudoscalar heavy mesons, SU(2) ChPT for kaons, and other relevant cases with the same pattern of spontaneous symmetry breaking and spinless matter fields.

The application of $\mathrm{SU}(2) \mathrm{ChPT}$ to kaon-pion scattering while treating the kaons as matter fields ensures a better convergence of chiral expansion than that of SU(3) ChPT which includes the kaons as Goldstone bosons as well [15. This theory is often used in the chiral extrapolation of lattice results at unphysical up and down quark masses to the physical values, see, e.g. Ref. [16].

While there are lots of data for the $K \pi$ scattering, no direct experimental data for $D \pi$

\footnotetext{
${ }^{1}$ Strictly speaking, the massless QCD Lagrangian has a $\mathrm{U}(N)_{L} \times \mathrm{U}(N)_{R}$ symmetry. However, the axial $\mathrm{U}(1)_{A}$ is broken at the quantum level due to the anomaly.
} 
scattering exists. Nevertheless, there have been lattice calculations of the scattering lengths for the scattering of a light pseudoscalar meson off a charmed meson [17 21]. The investigation of the $S$-wave scattering processes in the charmed $D$-meson sector is important for the understanding of the $0^{+}$strange and non-strange charmed mesons [18,22, 29]. The lattice results have been used to fix the LECs in the chiral Lagrangian for the $D$ mesons [29 33].

For purely Goldstone boson ChPT, the systematic one-loop renormalization has been done in the classical papers by Gasser and Leutwyler [2,3] using the background field method and heat kernel expansion techniques. The divergences in the one-loop effective action are calculated using dimensional regularization which preserves all the symmetries, and one finds all the counterterms which absorb these divergences and renormalize the theory at the order $\mathcal{O}\left(p^{4}\right)$. The complete renormalization of the two flavor heavy baryon chiral pion-nucleon Lagrangian is performed in Refs. 34, 35. Due to the consistent power counting of HBChPT, the UV divergences of the one-loop diagrams from the leading order Lagrangian only shift the LECs of the next-to-next-to-leading order, i.e., $\mathcal{O}\left(p^{3}\right)$. However, in relativistic baryonic ChPT (or ChPT including heavy mesons or matter-field kaons), the one-loop diagrams need to be renormalized by LECs from different orders.

In the present work, we employ the background field method and heat kernel techniques to study the renormalization of the chiral Lagrangian for scalar or pseudoscalar matter fields in the $\mathrm{SU}(N)$ fundamental representation to $\mathcal{O}\left(p^{3}\right) 2$ The paper is organized as follows. In Section 2, we collect the relevant notations and the Lagrangians. In Section 3, we derive the relevant one-loop effective action using the background field method and heat kernel techniques. The renormalization is carried out and a comparison with purely Goldstone boson ChPT is also given in Section 4. Section 5 presents a brief summary. Finally, we collect the explicit expressions for the "field strength" tensor and some useful identities in the Appendices $\mathrm{A}$ and $\mathrm{B}$, respectively, and relevant Lagrangian terms of the $\mathrm{SU}(2) \mathrm{ChPT}$ for kaons in Appendix C.

\section{Chiral Lagrangian of spinless matter fields}

In this section, we briefly review the chiral generating functional and Lagrangian describing the interaction between the Goldstone bosons and spinless matter fields, denoted by $\phi$ and $P$, respectively. The Goldstone bosons are encoded in an $N \times N$ unimodular, unitary matrix $U(x)$,

$$
U(x)=u^{2}(x)=\exp \left(i \frac{\phi}{F_{0}}\right)
$$

where $F_{0}$ is the pion decay constant in the chiral limit. $U(x)=u^{2}(x)=\exp \left(i \phi / F_{0}\right)$, and $\phi$ can be expanded in the $N \times N$ traceless Hermitian basis $\phi=\lambda^{a} \phi^{a}$ ( $\lambda^{a}$ denotes the Gell-Mann matrices for $N=3$ and the Pauli matrices for $N=2$ ) with $\phi^{a}$ being the corresponding Goldstone boson fields. We choose the representation in the coset space $\mathrm{SU}(N)_{L} \times \mathrm{SU}(N)_{R} / \mathrm{SU}(N)_{V}$ such that $U(x)$ and $u(x)$ transform under $\mathrm{SU}(N)_{L} \times \mathrm{SU}(N)_{R}$ as

$$
U \mapsto g_{L} U g_{R}^{\dagger}, \quad u \mapsto g_{L} u h^{\dagger}=h u g_{R}^{\dagger},
$$

where $g_{L} \in \mathrm{SU}(N)_{L}, g_{R} \in \mathrm{SU}(N)_{R}$, and the compensator field $h \in \mathrm{SU}(N)$ is a complicated nonlinear function of $g_{L}, g_{R}$ and $\phi$ which reduces to the element of the conserved

\footnotetext{
${ }^{2}$ For the case of scalar/pseudoscalar fields in the adjoint representations, the one-loop renormalization has been performed in Ref. [36].
} 
subgroup $\mathrm{SU}(N)_{V}$ when $g_{L}=g_{R}$. The transformation properties of the matter fields are not unique, but it is convenient to construct the matter fields such that they transform under $\mathrm{SU}(N)_{L} \times \mathrm{SU}(N)_{R}$ as

$$
P \mapsto P h^{\dagger}, \quad P^{\dagger} \mapsto h P^{\dagger} .
$$

This corresponds to the representation with $P^{\dagger}$ being in the fundamental representation of $\mathrm{SU}(N)_{V}$.

We will consider the case of the interaction between Goldstone bosons with a single matter field. The generating functional for the correlation functions of quark currents between single matter fields is defined via

$$
e^{i Z\left[j, J, J^{\dagger}\right]}=\mathcal{N} \int[d \phi][d P]\left[d P^{\dagger}\right] \exp i\left\{S_{\phi}+S_{\phi P}+\int d^{4} x\left(P J^{\dagger}+J P^{\dagger}\right)\right\},
$$

where $S_{\phi}=\int d^{4} x \mathcal{L}_{\phi}$ and $S_{\phi P}=\int d^{4} x \mathcal{L}_{\phi P}$ denote the Goldstone boson and the $\phi P$ chiral actions, respectively, $J$ and $J^{\dagger}$ are the sources coupled to the spinless matter fields, and $j$ denotes various external fields (vector $v_{\mu}$, axial $a_{\mu}$, scalar $s$ and pseudoscalar $p$ ). As usual, the quark mass terms will be included in the external scalar source $s$. The effective chiral Lagrangians can be expanded as

$$
\mathcal{L}_{\phi}=\sum_{n=1}^{\infty} \mathcal{L}_{\phi}^{(2 n)}, \quad \mathcal{L}_{\phi P}=\sum_{n=1}^{\infty} \mathcal{L}_{\phi P}^{(n)},
$$

where the superscripts denote the chiral dimension. We will consider the renormalization at the leading one-loop order, i.e. $\mathcal{O}\left(p^{3}\right)$ for the $\phi P$ part and $\mathcal{O}\left(p^{4}\right)$ for the purely Goldstone boson part 3

The leading order Goldstone boson Lagrangian reads

$$
\mathcal{L}_{\phi}^{(2)}=\frac{F_{0}^{2}}{4}\left\langle u_{\mu} u^{\mu}\right\rangle+\frac{F_{0}^{2}}{4}\left\langle\chi_{+}\right\rangle
$$

where $\langle\ldots\rangle$ denotes the trace in light-flavor space, and we use

$$
u_{\mu}=i\left(u^{\dagger} \nabla_{\mu}^{R} u-u \nabla_{\mu}^{L} u^{\dagger}\right), \quad \chi_{ \pm}=u^{\dagger} \chi u^{\dagger} \pm u \chi^{\dagger} u
$$

with the left and right covariant derivatives given by $\nabla_{\mu}^{R} u=\partial_{\mu} u-i r_{\mu} u$ and $\nabla_{\mu}^{L} u^{\dagger}=\partial_{\mu} u^{\dagger}-$ $i \ell_{\mu} u^{\dagger}$, where $r_{\mu}=v_{\mu}+a_{\mu}, l_{\mu}=v_{\mu}-a_{\mu}$ and $\chi=2 B_{0}(s+i p)$, with $B_{0}$ a positive constant related to the quark condensate. They transform under $\mathrm{SU}(N)_{L} \times \mathrm{SU}(N)_{R}$ as

$$
u_{\mu} \mapsto h u_{\mu} h^{\dagger}, \quad \chi_{ \pm} \mapsto h \chi_{ \pm} h^{\dagger} .
$$

The chiral effective Lagrangian for spinless matter fields starts from $\mathcal{O}(p)$, and the leading order terms are given by

$$
\mathcal{L}_{\phi P}^{(1)}=D_{\mu} P D^{\mu} P^{\dagger}-m^{2} P P^{\dagger},
$$

\footnotetext{
${ }^{3}$ One reason to include the purely Goldstone boson part to $\mathcal{O}\left(p^{4}\right)$ is due to the power counting rule that the Goldstone boson propagator $i /\left(q^{2}-m_{\phi}^{2}\right)$ is counted as $\mathcal{O}\left(p^{-2}\right)$, while the matter field propagator $i /\left(q^{2}-m^{2}\right)$ is counted as $\mathcal{O}\left(p^{-1}\right)$. As a result, the $\mathcal{O}\left(p^{4}\right)$ Goldstone boson Lagrangian could enter the calculation of the amplitudes for single matter fields of $\mathcal{O}\left(p^{3}\right)$. One example is given by the contribution of the wave function renormalization of the Goldstone bosons to the $\phi-P$ scattering amplitudes at $\mathcal{O}\left(p^{3}\right)[33$.
} 
where $m$ stands for the mass of the matter fields in the chiral limit, and the chirally covariant derivative acting on the matter fields are

$$
D_{\mu} P=\partial_{\mu} P+P \Gamma_{\mu}^{\dagger}, \quad D_{\mu} P^{\dagger}=\partial_{\mu} P^{\dagger}+\Gamma_{\mu} P^{\dagger}
$$

with the chiral connection $\Gamma_{\mu}=\frac{1}{2}\left(u^{\dagger} \nabla_{\mu}^{R} u+u \nabla_{\mu}^{L} u^{\dagger}\right)$. Under $\mathrm{SU}(N)_{L} \times \mathrm{SU}(N)_{R}, D_{\mu} P$ and $D_{\mu} P^{\dagger}$ transform in the same way as $P$ and $P^{\dagger}$, respectively.

For later use, we list the $\phi P$ Lagrangian up to $\mathcal{O}\left(p^{3}\right)$ [28, 33],

$$
\begin{aligned}
\mathcal{L}_{\phi P}^{(2)}= & P\left[-h_{0}\left\langle\chi_{+}\right\rangle-h_{1} \chi_{+}+h_{2}\left\langle u_{\mu} u^{\mu}\right\rangle-h_{3} u_{\mu} u^{\mu}\right] P^{\dagger} \\
& +D_{\mu} P\left[h_{4}\left\langle u_{\mu} u^{\nu}\right\rangle-h_{5}\left\{u^{\mu}, u^{\nu}\right\}\right] D_{\nu} P^{\dagger}, \\
\mathcal{L}_{\phi P}^{(3)}= & {\left[i g_{1} P\left[\chi_{-}, u_{\nu}\right] D^{\nu} P^{\dagger}+g_{2} P\left[u^{\mu}, \nabla_{\mu} u_{\nu}+\nabla_{\nu} u_{\mu}\right] D^{\nu} P^{\dagger}+g_{3} P\left[u_{\mu}, \nabla_{\nu} u_{\rho}\right] D^{\mu \nu \rho} P^{\dagger}\right.} \\
& \left.+g_{4} P \nabla_{\nu} \chi_{+} D^{\nu} P^{\dagger}+g_{5} P\left\langle\nabla_{\nu} \chi_{+}\right\rangle D^{\nu} P^{\dagger}+h . c .\right] \\
& +i \gamma_{1} D^{\mu} P f_{\mu \nu}^{+} D^{\nu} P^{\dagger}+\gamma_{2} P\left[u^{\mu}, f_{\mu \nu}^{-}\right] D^{\nu} P^{\dagger},
\end{aligned}
$$

where we have defined

$$
D^{\mu \nu \rho}=\left\{D_{\mu},\left\{D_{\nu}, D_{\rho}\right\}\right\}
$$

and

$$
f_{\mu \nu}^{ \pm}=u^{\dagger}\left(\partial_{\mu} \ell_{\nu}-\partial_{\nu} \ell_{\mu}-i\left[\ell_{\mu}, \ell_{\nu}\right]\right) u \pm u\left(\partial_{\mu} r_{\nu}-\partial_{\nu} r_{\mu}-i\left[r_{\mu}, r_{\nu}\right]\right) u^{\dagger}
$$

The $\gamma_{1}$ and $\gamma_{2}$ terms have not been introduced in the literature to the best of our knowledge. Although they do not contribute to the scattering, they are necessary for renormalization as one can see later.

For the two-flavor case $N=2$, one may use the Caylay-Hamilton relation, see Eq. (35), to reduce the number of terms. Since the Lagrangian in this case has been constructed in Ref. [15], we give the relations between our LECs and the ones therein $\left(A_{i}, B_{i}\right.$ and $\left.C_{i}\right)$ (for completeness, we copy in Appendix C the relevant Lagrangian terms from Ref. [15])

$$
\begin{aligned}
& A_{1}=2 h_{3}-4 h_{2}, \quad A_{2}=4\left(h_{5}-h_{4}\right), \quad A_{3}=-h_{1}, \quad A_{4}=-h_{0}, \\
& B_{1}=8 g_{2}, \quad B_{3}=-2 g_{1}, \\
& C_{3}=8 g_{3}, \quad C_{5}=-2 g_{4}, \quad C_{6}=-2 g_{5} .
\end{aligned}
$$

Notice that we do not have the $B_{2}$ term here because it is in fact a $\mathcal{O}\left(p^{4}\right)$ term as can be seen by partial integration. Instead, part of the $C_{3}$ term in the $\mathcal{O}\left(p^{4}\right)$ Lagrangian of Ref. [15], the second line of the $C_{3}$ term in Eq. (39), is in fact of $\mathcal{O}\left(p^{3}\right)$, and the relation given above is derived by keeping only that part in the $C_{3}$ term. These corrected assignments can be checked from the explicit expression of the isospin-3/2 $\pi K$ scattering amplitude in Ref. [37. The $g_{5}$ and $g_{6}$ terms are not listed in our original Lagrangian [33] because their contribution to the scattering amplitudes get cancelled by the wave function renormalization of the matter field at the order $\mathcal{O}\left(p^{3}\right)$. However, we list them since they are formally of $\mathcal{O}\left(p^{3}\right)$, and they can be rewritten as the $C_{5}$ and $C_{6}$ terms in Ref. [15] by using the equation of motion for the matter field. 


\section{Generating functional to one-loop}

In this section, we evaluate the generating functional to the leading one-loop order using the background field method. The basic idea of the background field method is to decompose the fields into classical background fields and quantum fluctuations. After integrating out the fluctuations, the resulting effective action actually describes the one-loop contribution of the original action. If only the divergent parts of the loops are considered, one could employ the heat kernel techniques to extract the UV divergence of the effective action, which contains all the possible one-loop divergences and needs to be renormalized by various counterterms provided by the LECs of the higher order Lagrangians. To this end, we perturb the fields $u(x)$ and $P(x)$ around the solutions of the classical equations of motion $\bar{u}(x)$ and $\bar{P}(x)$,

$$
\begin{aligned}
u^{2} & =\bar{u} e^{-i \eta} \bar{u}, \\
P & =\bar{P}+h,
\end{aligned}
$$

where $\eta$ is a traceless Hermitian matrix, $\eta=\eta^{a} \lambda^{a}\left(a=1, \ldots, N^{2}-1\right)$. Then we substitute the decompositions in Eq. (15) into the generating functional given by Eq. (4). Since we work up to the leading one-loop order, we retain only the quadratic terms in $\eta$ and $h$ from $\mathcal{L}_{\phi}^{(2)}$ and $\mathcal{L}_{\phi P}^{(1)}$ while the terms linear in the fluctuations give the equations of motion, see Appendix B. For convenience, we collect the fluctuations in the following vectors

$$
\xi_{A}=\left(\eta^{a}, \frac{\sqrt{2}}{F_{0}} h_{i}\right), \quad \xi_{B}^{\dagger}=\left(\eta^{b}, \frac{\sqrt{2}}{F_{0}} h_{j}^{\dagger}\right)^{T},
$$

where $i, j=1, \ldots, N$ while $A$ and $B$ run from 1 to $\left(N^{2}-1\right)+N$ for $N^{2}-1$ Goldstone boson fluctuations and $N$ matter field fluctuations. To second order in the fluctuations, the chiral connection $\Gamma_{\mu}$, the axial-vector vielbein $u_{\mu}$ and $\chi_{+}$read

$$
\begin{aligned}
\Gamma_{\mu} & =\bar{\Gamma}_{\mu}+\frac{1}{4}\left[\bar{u}_{\mu}, \eta\right]+\frac{1}{8}\left[\eta, \nabla_{\mu} \eta\right]+\mathcal{O}\left(\eta^{3}\right), \\
\nabla_{\mu} \eta & =\partial_{\mu} \eta+\left[\bar{\Gamma}_{\mu}, \eta\right] \\
u_{\mu} & =\bar{u}_{\mu}-\nabla_{\mu} \eta+\frac{1}{8}\left[\eta,\left[\bar{u}_{\mu}, \eta\right]\right]+\mathcal{O}\left(\eta^{3}\right), \\
\chi_{+} & =\bar{\chi}_{+}-\frac{i}{2}\left\{\bar{\chi}_{-}, \eta\right\}-\frac{1}{8}\left\{\eta,\left\{\bar{\chi}_{+}, \eta\right\}\right\}+\mathcal{O}\left(\eta^{3}\right) .
\end{aligned}
$$

From now on, we will neglect the bars over the classical field configurations for brevity. Using the expressions in Eq. (17), the terms in the action quadratic in $\xi$ take the form of

$$
S^{\text {quad }}=-\frac{F_{0}^{2}}{2} \int d x \xi_{A}\left(\mathbb{D}_{\mu} \mathbb{D}^{\mu}+\sigma\right)^{A B} \xi_{B}^{\dagger} .
$$

Here, the covariant derivative $\mathbb{D}_{\mu}^{A B}$ is given in matrix form by

$$
\mathbb{D}_{\mu}^{A B}=\left(\begin{array}{cc}
d_{\mu}^{a b} & \frac{1}{4 \sqrt{2} F_{0}}\left(P\left[u_{\mu}, \lambda^{a}\right]\right)_{j} \\
\frac{1}{4 \sqrt{2} F_{0}}\left(\left[u_{\mu}, \lambda^{b}\right] P^{\dagger}\right)_{i} & D_{\mu}^{i j}
\end{array}\right)
$$


where

$$
\begin{aligned}
d_{\mu}^{a b} & =\delta^{a b} \partial_{\mu}-\frac{1}{2}\left\langle\left[\lambda^{a}, \lambda^{b}\right] \Gamma_{\mu}\right\rangle-\frac{1}{8 F_{0}^{2}}\left(D_{\mu} P\left[\lambda^{a}, \lambda^{b}\right] P^{\dagger}-P\left[\lambda^{a}, \lambda^{b}\right] D_{\mu} P^{\dagger}\right), \\
D_{\mu}^{i j} & =\delta^{i j} \partial_{\mu}+\left(\Gamma_{\mu}\right)^{i j},
\end{aligned}
$$

and the non-derivative term $\sigma^{A B}$ stands for

$$
\sigma^{A B}=\left(\begin{array}{ll}
\sigma_{11}^{a b} & \sigma_{12}^{a j} \\
\sigma_{21}^{i b} & \sigma_{22}^{i j}
\end{array}\right),
$$

with

$$
\begin{aligned}
\sigma_{11}^{a b}= & -\frac{1}{8}\left\langle u_{\mu}\left[\lambda^{a},\left[u^{\mu}, \lambda^{b}\right]\right]\right\rangle+\frac{1}{16}\left\langle\left\{\lambda^{a},\left\{\chi_{+}, \lambda^{b}\right\}\right\}\right\rangle+\frac{3}{32 F_{0}^{2}} P\left[u_{\mu}, \lambda^{a}\right]\left[u^{\mu}, \lambda^{b}\right] P^{\dagger} \\
& -\frac{1}{64 F_{0}^{4}}\left(D_{\mu} P\left[\lambda^{a}, \lambda^{c}\right] P^{\dagger}-P\left[\lambda^{a}, \lambda^{c}\right] D_{\mu} P^{\dagger}\right)\left(D^{\mu} P\left[\lambda^{c}, \lambda^{b}\right] P^{\dagger}-P\left[\lambda^{c}, \lambda^{b}\right] D^{\mu} P^{\dagger}\right), \\
\sigma_{12}^{a j}= & -\frac{1}{4 \sqrt{2} F_{0}}\left(P\left[\nabla_{\mu} u^{\mu}, \lambda^{a}\right]\right)_{j}-\frac{3}{4 \sqrt{2} F_{0}}\left(D_{\mu} P\left[u^{\mu}, \lambda^{a}\right]\right)_{j}, \\
& +\frac{1}{32 \sqrt{2} F_{0}^{3}}\left(D_{\mu} P\left[\lambda^{a}, \lambda^{c}\right] P^{\dagger}-P\left[\lambda^{a}, \lambda^{c}\right] D_{\mu} P^{\dagger}\right)\left(P\left[u_{\mu}, \lambda^{c}\right]\right)_{j} \\
\sigma_{21}^{i b}= & \frac{1}{4 \sqrt{2} F_{0}}\left(\left[\nabla_{\mu} u^{\mu}, \lambda^{b}\right] P^{\dagger}\right)_{i}+\frac{3}{4 \sqrt{2} F_{0}}\left(\left[u^{\mu}, \lambda^{b}\right] D_{\mu} P^{\dagger}\right)_{i}, \\
& +\frac{1}{32 \sqrt{2} F_{0}^{3}}\left(\left[u_{\mu}, \lambda^{c}\right] P^{\dagger}\right)_{i}\left(D_{\mu} P\left[\lambda^{c}, \lambda^{b}\right] P^{\dagger}-P\left[\lambda^{c}, \lambda^{b}\right] D_{\mu} P^{\dagger}\right) \\
\sigma_{22}^{i j}= & m^{2} \delta^{i j}-\frac{1}{32 F_{0}^{2}}\left(\left[u_{\mu}, \lambda^{c}\right] P^{\dagger}\right)_{i}\left(P\left[u^{\mu}, \lambda^{c}\right]\right)_{j} .
\end{aligned}
$$

In each of the $\sigma_{11,12,21}$, the last term contains more than two matter fields, and thus does not contribute to the correlation function of operators sandwiched between single matter fields. The one-loop term in the generating functional is a Gaussian integral over the fluctuations $\xi$, which can be evaluated with standard methods [38, 39]:

$$
\begin{aligned}
e^{i Z_{\text {loop }}} & =\int[d \xi] \exp \left\{-i \frac{F_{0}^{2}}{2} \xi_{A}\left(\mathbb{D}_{\mu} \mathbb{D}^{\mu}+\sigma\right)^{A B} \xi_{B}^{\dagger}\right\} \\
& =\mathcal{N}\left(\operatorname{det}\left[\mathbb{D}_{\mu} \mathbb{D}^{\mu}+\sigma\right]\right)^{-1 / 2} \\
& =\mathcal{N} \exp \left\{-\frac{1}{2} \operatorname{tr} \log \left(\mathbb{D}_{\mu} \mathbb{D}^{\mu}+\sigma\right)\right\}
\end{aligned}
$$

where $\mathcal{N}$ is an irrelevant normalization, "tr" stands for the trace over the space-time as well as the flavor space spanned by the basis of the $\xi_{A}$. The UV divergences in the generating functional $Z_{\text {loop }}$ can be extracted by using the heat kernel expansion (see, e.g., Appendix B of Ref. [38]), and they only show up in the first few expansion coefficients. Using dimensional 
regularization, the relevant terms are

$$
\begin{aligned}
Z_{\text {loop }}= & \frac{i}{2} \operatorname{tr} \log \left(\mathbb{D}_{\mu} \mathbb{D}^{\mu}+\sigma\right) \\
= & \frac{1}{2(4 \pi)^{d / 2}} \int d^{4} x\left[\Gamma\left(1-\frac{d}{2}\right) \mu^{d-2} \operatorname{Tr}(\sigma)\right. \\
& \left.+\mu^{d-4} \Gamma\left(2-\frac{d}{2}\right) \operatorname{Tr}\left(\frac{1}{12} \mathbb{F}_{\mu \nu} \mathbb{F}^{\mu \nu}+\frac{1}{2} \sigma^{2}\right)+\ldots\right],
\end{aligned}
$$

where "Tr" denotes the trace over the space spanned by the $\xi_{A}$, and the associated "field strength" tensor $\mathbb{F}_{\mu \nu}^{A B}=\left[\mathbb{D}_{\mu}, \mathbb{D}_{\nu}\right]^{A B}$ is given in Appendix $\mathbb{A}$. Further, $\mu$ denotes the scale of dimensional regularization. Only the second term, the $1 /(d-4)$ pole, contributes to the UV divergences at $d=4$.

\section{Renormalization}

As we are only interested in the UV divergences, we substitute the explicit expressions of $\mathbb{F}$ and $\sigma$ into Eq. (24) and keep only the terms having a $1 /(d-4)$ pole. Using the equations of motion for the classical fields and the identities listed in Appendix B. one can obtain all possible one-loop divergences. Up to $\mathcal{O}\left(p^{3}\right)$, those relevant for single matter fields read

$$
\begin{aligned}
Z_{\phi P}^{\operatorname{div}}= & -\frac{\lambda}{F_{0}^{2}} \int d^{d} x\left[\frac{m^{2}}{24} P\left\langle u^{\mu} u_{\mu}\right\rangle P^{\dagger}+\frac{m^{2}}{24} N P u^{\mu} u_{\mu} P^{\dagger}+\frac{7}{12} D_{\mu} P\left\langle u^{\mu} u^{\nu}\right\rangle D_{\nu} P^{\dagger}\right. \\
& +\frac{7}{24} N D_{\mu} P\left\{u^{\mu}, u^{\nu}\right\} D_{\nu} P^{\dagger}-\frac{3}{64} N\left(P\left[u^{\mu}, \nabla_{\mu} u_{\nu}+\nabla_{\nu} u_{\mu}\right] D^{\nu} P^{\dagger}+\text { h.c. }\right) \\
& \left.+\frac{N}{6} i D^{\mu} P f_{\mu \nu}^{+} D^{\nu} P^{\dagger}+\frac{11}{96} N P\left[u^{\mu}, f_{\mu \nu}^{-}\right] D^{\nu} P^{\dagger}\right]
\end{aligned}
$$

where $\lambda=\mu^{d-4}\left[(4 \pi)^{d / 2}(d-4)\right]^{-1}$.

In order to obtain a finite one-loop effective action, the UV divergences in Eq. (24) needs to be cancelled by those of the LECs

$$
\mathcal{L}=\sum_{i} c_{i} \mathcal{O}_{i}=\sum_{i}\left[c_{i}^{r}(\mu)+c_{i}^{0} \lambda\right] \mathcal{O}_{i}
$$

where $c_{i}^{r}(\mu)$ is the finite part of the $c_{i}$ and is scale dependent. From Eq. (25) and Eq. (30),

it is easy to read off the divergent parts of the corresponding LECs, which up to $\mathcal{O}\left(p^{3}\right)$ are given by

$$
\begin{array}{r}
h_{0}^{0}=h_{1}^{0}=0, \quad h_{2}^{0}=\frac{m^{2}}{24}, \quad h_{3}^{0}=-\frac{m^{2}}{24} N, \quad h_{4}^{0}=\frac{7}{12}, \quad h_{5}^{0}=-\frac{7}{24} N, \\
g_{1}^{0}=0, \quad g_{2}^{0}=-\frac{3}{64} N, \quad g_{3,4,5}^{0}=0, \quad \gamma_{1}^{0}=\frac{N}{6}, \quad \gamma_{2}^{0}=\frac{11}{96} N .
\end{array}
$$

These coefficients determine the scale dependence of the corresponding renormalized LECs, and the pertinent renormalization group equations read

$$
\frac{\partial c_{i}^{r}(\mu)}{\partial \mu}=-\frac{c_{i}^{0}}{16 \pi^{2}}
$$


From Eq. (27), once clearly sees that the one-loop divergences also renormalize the LECs at a lower order (here, the $h_{i}$ 's). For $N=3$, the values of $h_{i}^{0}$ and $g_{i}^{0}$ agree with those found in an explicit calculation of the scattering amplitudes [33].

We have checked that integrating out the Goldstone boson fluctuations leads to the divergence structure of the purely Goldstone boson effective action for $N$ flavors [40]:

$$
\begin{aligned}
Z_{\phi}^{\operatorname{div}, \phi}= & -\lambda \int d^{d} x\left[\frac{N}{48}\left\langle u_{\mu} u_{\nu} u^{\mu} u^{\nu}\right\rangle+\frac{1}{16}\left\langle u_{\mu} u^{\mu}\right\rangle^{2}+\frac{1}{8}\left\langle u_{\mu} u_{\nu}\right\rangle\left\langle u^{\mu} u^{\nu}\right\rangle\right. \\
& +\frac{N}{24}\left\langle u_{\mu} u^{\mu} u_{\nu} u^{\nu}\right\rangle+\frac{1}{8}\left\langle u_{\mu} u^{\mu}\right\rangle\left\langle\chi_{+}\right\rangle+\frac{N}{8}\left\langle u_{\mu} u^{\mu} \chi_{+}\right\rangle+\frac{N^{2}+2}{16 N^{2}}\left\langle\chi_{+}\right\rangle^{2} \\
& \left.+\frac{N^{2}-4}{16 N}\left\langle\chi_{+}^{2}\right\rangle-\frac{N}{12} i\left\langle f_{\mu \nu}^{+} u^{\mu} u^{\nu}\right\rangle-\frac{N}{24}\left\langle f_{\mu \nu}^{+} f^{+\mu \nu}\right\rangle\right] .
\end{aligned}
$$

Yet, as can be expected, $Z_{\phi}^{\text {div }}$ also gets contributions due to the presence of the matter field loops. The matter fields are expected to be much heavier than the Goldstone bosons, and therefore their effects in loops on the properties of Goldstone bosons might be irrelevant, at least for the ChPT of QCD. Nevertheless, they contribute to the divergence of the generational functional, which is given here for completeness

$$
Z_{\phi}^{\operatorname{div}, P}=-\lambda \int d^{d} x\left[\frac{1}{96}\left\langle u_{\mu} u_{\nu} u^{\mu} u^{\nu}\right\rangle-\frac{1}{96}\left\langle u_{\mu} u^{\mu} u_{\nu} u^{\nu}\right\rangle-\frac{i}{24}\left\langle f_{\mu \nu}^{+} u^{\mu} u^{\nu}\right\rangle-\frac{1}{48}\left\langle f_{\mu \nu}^{+} f^{+\mu \nu}\right\rangle\right] .
$$

\section{Summary}

In this paper, we have performed the renormalization of the chiral Lagrangian for spinless matter fields living in the fundamental representation of $\mathrm{SU}(N)$. For QCD, the matter fields can be kaons for $\mathrm{SU}(2)$ kaon ChPT or pseudoscalar heavy mesons. Yet, it can also be applied to any other theory with a spontaneous symmetry breaking of $\mathrm{SU}(N)_{L} \times \mathrm{SU}(N)_{R}$ to $\mathrm{SU}(N)_{V}$ and spinless matter fields in the fundamental representation. Using the background field method and heat kernel expansion techniques, we calculated the divergence of the oneloop effective generating functional for correlation functions of single matter fields up to the order $\mathcal{O}\left(p^{3}\right)$, which can be absorbed by various LECs in both the $\mathcal{O}\left(p^{2}\right)$ and $\mathcal{O}\left(p^{3}\right)$ Lagrangians.

\section{Acknowledgements}

We thank Bastian Kubis and Akaki Rusetsky for useful discussions. FKG gratefully acknowledges the hospitality at the HISKP where part of this work was done. This work is supported in part by DFG and NSFC through funds provided to the Sino-German CRC 110 "Symmetries and the Emergence of Structure in QCD" (NSFC Grant No. 11261130311). FKG is also supported by the Thousand Talents Plan for Young Professionals. The work of UGM was supported in part by The Chinese Academy of Sciences (CAS) President's International Fellowship Initiative (PIFI) with grant no. 2015VMA076. 


\section{A The "field-strength" tensor}

The "field-strength" tensor in Eq. (24) is

$$
\mathbb{F}_{\mu \nu}^{A B}=\left(\begin{array}{cc}
-\frac{1}{2}\left\langle\left[\lambda^{a}, \lambda^{b}\right] \mathrm{A}_{\mu \nu}\right\rangle+\Sigma_{11}^{a b} & \Sigma_{12}^{a j} \\
\Sigma_{21}^{i b} & \Gamma_{\mu \nu}^{i j}+\Sigma_{22}^{i j}
\end{array}\right),
$$

where 4

$$
\begin{aligned}
\mathrm{A}_{\mu \nu}= & \Gamma_{\mu \nu}+\frac{1}{4 F_{0}^{2}}\left(2 D_{\mu} P^{\dagger} D_{\nu} P-2 D_{\nu} P^{\dagger} D_{\mu} P+P^{\dagger}\left[D_{\mu}, D_{\nu}\right] P-\left[D_{\mu}, D_{\nu}\right] P^{\dagger} P\right) \\
& +\frac{1}{\left(4 F_{0}^{2}\right)^{2}}\left[P^{\dagger} D_{\mu} P-D_{\mu} P^{\dagger} P, P^{\dagger} D_{\nu} P-D_{\nu} P^{\dagger} P\right], \\
\Sigma_{11}^{a b}= & \frac{1}{32 F_{0}^{2}}\left(P\left[u_{\mu}, \lambda^{a}\right]\left[u_{\nu}, \lambda^{b}\right] P^{\dagger}-P\left[u_{\nu}, \lambda^{a}\right]\left[u_{\mu}, \lambda^{b}\right] P^{\dagger}\right), \\
\Sigma_{12}^{a j}= & \frac{1}{4 \sqrt{2} F_{0}}\left(D_{\mu} P\left[u_{\nu}, \lambda^{a}\right]-D_{\nu} P\left[u_{\mu}, \lambda^{a}\right]+P\left[\nabla_{\mu} u_{\nu}-\nabla_{\nu} u_{\mu}, \lambda^{a}\right]\right)_{j} \\
& -\frac{1}{32 \sqrt{2} F_{0}^{3}}\left[\left(D_{\mu} P\left[\lambda^{a}, \lambda^{c}\right] P^{\dagger}-P\left[\lambda^{a}, \lambda^{c}\right] D_{\mu} P^{\dagger}\right)\left(P\left[u_{\nu}, \lambda^{c}\right]\right)_{j}-(\mu \leftrightarrow \nu)\right], \\
\Sigma_{21}^{i b}= & \frac{1}{4 \sqrt{2} F_{0}}\left(\left[u_{\nu}, \lambda^{b}\right] D_{\mu} P^{\dagger}-\left[u_{\mu}, \lambda^{b}\right] D_{\nu} P^{\dagger}+\left[\nabla_{\mu} u_{\nu}-\nabla_{\nu} u_{\mu}, \lambda^{b}\right] P^{\dagger}\right)_{i} \\
& -\frac{1}{32 \sqrt{2} F_{0}^{3}}\left[\left(\left[u_{\mu}, \lambda^{c}\right] P^{\dagger}\right)_{i}\left(D_{\nu} P\left[\lambda^{c}, \lambda^{b}\right] P^{\dagger}-P\left[\lambda^{c}, \lambda^{b}\right] D_{\nu} P^{\dagger}\right)-(\mu \leftrightarrow \nu)\right], \\
\Sigma_{22}^{i j}= & \frac{1}{32 F_{0}^{2}}\left[\left(\left[u_{\mu}, \lambda^{c}\right] P^{\dagger}\right)_{i}\left(P\left[u_{\nu}, \lambda^{c}\right]\right)_{j}-\left(\left[u_{\nu}, \lambda^{c}\right] P^{\dagger}\right)_{i}\left(P\left[u_{\mu}, \lambda^{c}\right]\right)_{j}\right],
\end{aligned}
$$

with

$$
\Gamma_{\mu \nu}=\left[D_{\mu}, D_{\nu}\right]=\partial_{\mu} \Gamma_{\nu}-\partial_{\nu} \Gamma_{\mu}+\left[\Gamma_{\mu}, \Gamma_{\nu}\right]
$$

\section{B Some useful identities}

The equations of motion of the classical fields and some useful identities are collected here:

$$
\begin{aligned}
& \nabla_{\mu} u^{\mu}=\frac{i}{2}\left(\chi_{-}-\frac{1}{N}\left\langle\chi_{-}\right\rangle\right), \\
& D_{\mu} D^{\mu} P^{\dagger}+m^{2} P^{\dagger}=0, \\
& \Gamma_{\mu \nu}=\left[D_{\mu}, D_{\nu}\right]=\frac{1}{4}\left[u_{\mu}, u_{\nu}\right]-\frac{i}{2} f_{\mu \nu}^{+}, \\
& \nabla_{\mu} u_{\nu}-\nabla_{\nu} u_{\mu}=-f_{\mu \nu}^{-} .
\end{aligned}
$$

The Cayley-Hamilton theorem states that every square matrix over a commutative ring satisfies its own characteristic equation. For the two-dimensional case, the theorem implies the relation

$$
\{A, B\}=A\langle B\rangle+B\langle A\rangle+\langle A B\rangle-\langle A\rangle\langle B\rangle,
$$

\footnotetext{
${ }^{4}$ Note that $P$ is a $1 \times N$ vector, and $P^{\dagger}$ is a $N \times 1$ vector. As a result, $P^{\dagger} P$ is a $N \times N$ matrix.
} 
for arbitrary $2 \times 2$ matrices $A$ and $B$. For the $\mathrm{SU}(3)$ case, we have

$$
\left\langle u^{\mu} u^{\nu} u_{\mu} u_{\nu}\right\rangle=-2\left\langle u^{\mu} u_{\mu} u^{\nu} u_{\nu}\right\rangle+\frac{1}{2}\left\langle u_{\mu} u^{\mu}\right\rangle^{2}+\left\langle u^{\mu} u_{\nu}\right\rangle^{2} .
$$

Two relations used in deriving Eq. (25) are

$$
\begin{aligned}
& D^{\mu} P\left\{u^{\nu}, \nabla_{\mu} u_{\nu}\right\} P^{\dagger}+P\left\{u^{\nu}, \nabla_{\mu} u_{\nu}\right\} D^{\mu} P^{\dagger} \\
= & D^{\mu} P\left\{u^{\nu}, \nabla_{\mu} u_{\nu}\right\} P^{\dagger}-D^{\mu} P\left\{u^{\nu}, \nabla_{\mu} u_{\nu}\right\} P^{\dagger}+\mathcal{O}\left(p^{4}\right) \\
= & \mathcal{O}\left(p^{4}\right)
\end{aligned}
$$

and

$$
\begin{aligned}
& D_{\mu} P\left[u^{\mu}, u^{\nu}\right] D_{\nu} P^{\dagger} \\
= & -P\left[\nabla_{\mu} u^{\mu}, u^{\nu}\right] D_{\nu} P^{\dagger}-P\left[u^{\mu}, \nabla_{\mu} u^{\nu}\right] D_{\nu} P^{\dagger}-P\left[u^{\mu}, u^{\nu}\right] D_{\mu} D_{\nu} P^{\dagger} \\
= & -\frac{1}{2}\left(P\left[u^{\mu}, \nabla_{\mu} u^{\nu}+\nabla_{\nu} u^{\mu}\right] D_{\nu} P^{\dagger}+P\left[u^{\mu}, \nabla_{\mu} u^{\nu}-\nabla_{\nu} u^{\mu}\right] D_{\nu} P^{\dagger}\right. \\
& \left.+P\left[u^{\mu}, u^{\nu}\right]\left[D_{\mu}, D_{\nu}\right] P^{\dagger}+2 P\left[\nabla_{\mu} u^{\mu}, u^{\nu}\right] D_{\nu} P^{\dagger}\right) \\
= & -\frac{1}{2}\left(P\left[u^{\mu}, \nabla_{\mu} u_{\nu}+\nabla_{\nu} u_{\mu}\right] D^{\nu} P^{\dagger}-P\left[u^{\mu}, f_{\mu \nu}^{-}\right] D^{\nu} P^{\dagger}\right. \\
& \left.+i P\left[\chi_{-}, u_{\nu}\right] D^{\nu} P^{\dagger}+\frac{1}{4} P\left[u^{\mu}, u^{\nu}\right]\left[u_{\mu}, u_{\nu}\right] P^{\dagger}-\frac{i}{2} P\left[u^{\mu}, u^{\nu}\right] f_{\mu \nu}^{+} P^{\dagger}\right) \\
= & -\frac{1}{2}\left(P\left[u^{\mu}, \nabla_{\mu} u_{\nu}+\nabla_{\nu} u_{\mu}\right] D^{\nu} P^{\dagger}-P\left[u^{\mu}, f_{\mu \nu}^{-}\right] D^{\nu} P^{\dagger}+i P\left[\chi_{-}, u_{\nu}\right] D^{\nu} P^{\dagger}\right) \\
& +\mathcal{O}\left(p^{4}\right) .
\end{aligned}
$$

\section{Relevant Lagrangian terms in kaon SU(2) ChPT}

Here we list the terms from Ref. [15] relevant for our comparison:

$$
\begin{aligned}
\mathcal{L}_{\pi K}^{(1)}= & D_{\mu} K^{\dagger} D^{\mu} K-M_{K}^{2} K^{\dagger} K, \\
\mathcal{L}_{\pi K}^{(2)}= & A_{1}\left\langle\Delta_{\mu} \Delta^{\mu}\right\rangle K^{\dagger} K+A_{2}\left\langle\Delta^{\mu} \Delta^{\nu}\right\rangle D_{\mu} K^{\dagger} D^{\nu} K+A_{3} K^{\dagger} \chi_{+} K+A_{4}\left\langle\chi_{+}\right\rangle K^{\dagger} K \\
\mathcal{L}_{\pi K}^{(3)}= & B_{1}\left(K^{\dagger}\left[\Delta^{\nu \mu}, \Delta_{\nu}\right] D_{\mu} K-D_{\mu} K^{\dagger}\left[\Delta^{\nu \mu}, \Delta_{\nu}\right] K\right) \\
& +B_{2}\left\langle\Delta^{\mu \nu} \Delta^{\rho}\right\rangle\left(D_{\mu \nu} K^{\dagger} D_{\rho} K+D_{\rho} K^{\dagger} D_{\mu \nu} K\right) \\
& +B_{3}\left(K^{\dagger}\left[\Delta_{\mu}, \chi_{-}\right] D^{\mu} K-D_{\mu} K^{\dagger}\left[\Delta^{\mu}, \chi_{-}\right] K\right), \\
\mathcal{L}_{\pi K}^{(4)}= & C_{3}\left[\left\langle\Delta^{\mu \nu} \Delta^{\rho}\right\rangle\left(D_{\mu \nu} K^{\dagger} D_{\rho} K+D_{\rho} K^{\dagger} D_{\mu \nu} K\right)\right. \\
& \left.\quad-2\left(D^{\mu \nu} K^{\dagger} \Delta_{\mu} \Delta_{\nu \rho} D^{\rho} K+D^{\rho} K^{\dagger} \Delta_{\nu \rho} \Delta_{\mu} D^{\mu \nu} K\right)\right] \\
& +C_{5}\left(D_{\mu} K^{\dagger} \chi_{+} D^{\mu} K-M_{K}^{2} K^{\dagger} \chi_{+} K\right)+C_{6}\left\langle\chi_{+}\right\rangle\left(D_{\mu} K^{\dagger} D^{\mu} K-M_{K}^{2} K^{\dagger} K\right) \\
& +\ldots
\end{aligned}
$$

The comparison can be performed by comparing notations in Ref. [15] with ours:

$\Delta_{\mu}=-\frac{i}{2} u_{\mu} \quad \Delta_{\mu \nu}=-\frac{i}{4}\left(\nabla_{\mu} u_{\nu}+\nabla_{\nu} u_{\mu}\right), \quad K^{\dagger}=P, \quad K=P^{\dagger}, \quad D_{\mu \nu}=\left\{D_{\mu}, D_{\nu}\right\}$. 


\section{References}

[1] S. Weinberg, Physica A 96, 327 (1979).

[2] J. Gasser and H. Leutwyler, Annals Phys. 158, 142 (1984).

[3] J. Gasser and H. Leutwyler, Nucl. Phys. B 250, 465 (1985).

[4] J. Gasser, M. E. Sainio and A. Švarc, Nucl. Phys. B 307, 779 (1988).

[5] E. E. Jenkins and A. V. Manohar, Phys. Lett. B 255, 558 (1991).

[6] V. Bernard, N. Kaiser, J. Kambor and U.-G. Meißner, Nucl. Phys. B 388, 315 (1992).

[7] H. B. Tang, hep-ph/9607436.

[8] P. J. Ellis and H. B. Tang, Phys. Rev. C 57, 3356 (1998) hep-ph/9709354].

[9] T. Becher and H. Leutwyler, Eur. Phys. J. C 9, 643 (1999) hep-ph/9901384.

[10] J. Gegelia and G. Japaridze, Phys. Rev. D 60, 114038 (1999) hep-ph/9908377.

[11] T. Fuchs, J. Gegelia, G. Japaridze and S. Scherer, Phys. Rev. D 68, 056005 (2003) hep-ph/0302117.

[12] G. Burdman and J. F. Donoghue, Phys. Lett. B 280, 287 (1992).

[13] M. B. Wise, Phys. Rev. D 45, 2188 (1992).

[14] T. M. Yan, H. Y. Cheng, C. Y. Cheung, G. L. Lin, Y. C. Lin and H. L. Yu, Phys. Rev. D 46, 1148 (1992) [Phys. Rev. D 55, 5851 (1997)].

[15] A. Roessl, Nucl. Phys. B 555, 507 (1999) hep-ph/9904230.

[16] C. Allton et al. [RBC-UKQCD Collaboration], Phys. Rev. D 78, 114509 (2008) arXiv:0804.0473 [hep-lat]].

[17] L. Liu, H. W. Lin and K. Orginos, PoS LATTICE 2008, 112 (2008) arXiv:0810.5412 [hep-lat]].

[18] L. Liu, K. Orginos, F.-K. Guo, C. Hanhart and U.-G. Meißner, Phys. Rev. D 87, 014508 (2013) arXiv:1208.4535 [hep-lat]].

[19] D. Mohler, S. Prelovsek and R. M. Woloshyn, Phys. Rev. D 87, 034501 (2013) arXiv:1208.4059 [hep-lat]].

[20] D. Mohler, C. B. Lang, L. Leskovec, S. Prelovsek and R. M. Woloshyn, Phys. Rev. Lett. 111, 222001 (2013) [arXiv:1308.3175 [hep-lat]].

[21] C. B. Lang, L. Leskovec, D. Mohler, S. Prelovsek and R. M. Woloshyn, Phys. Rev. D 90, 034510 (2014) arXiv:1403.8103 [hep-lat]].

[22] E. E. Kolomeitsev and M. F. M. Lutz, Phys. Lett. B 582 (2004) 39 hep-ph/0307133.

[23] J. Hofmann and M. F. M. Lutz, Nucl. Phys. A 733 (2004) 142 hep-ph/0308263. 
[24] F.-K. Guo, P.-N. Shen, H.-C. Chiang, R.-G. Ping and B.-S. Zou, Phys. Lett. B 641 (2006) 278 hep-ph/0603072].

[25] D. Gamermann, E. Oset, D. Strottman and M. J. Vicente Vacas, Phys. Rev. D 76 (2007) 074016 [hep-ph/0612179].

[26] A. Faessler, T. Gutsche, V. E. Lyubovitskij and Y. L. Ma, Phys. Rev. D 76, 014005 (2007) arXiv:0705.0254 [hep-ph]].

[27] M. F. M. Lutz and M. Soyeur, Nucl. Phys. A 813, 14 (2008) arXiv:0710.1545 [hep-ph]].

[28] F.-K. Guo, C. Hanhart, S. Krewald and U.-G. Meißner, Phys. Lett. B 666, 251 (2008) arXiv:0806.3374 [hep-ph]].

[29] F.-K. Guo, C. Hanhart and U.-G. Meißner, Eur. Phys. J. A 40, 171 (2009) arXiv:0901.1597 [hep-ph]].

[30] Y.-R. Liu, X. Liu and S.-L. Zhu, Phys. Rev. D 79, 094026 (2009) arXiv:0904.1770 [hep$\mathrm{ph}]$.

[31] L.-S. Geng, N. Kaiser, J. Martin-Camalich and W. Weise, Phys. Rev. D 82, 054022 (2010) arXiv:1008.0383 [hep-ph]].

[32] P. Wang and X. G. Wang, Phys. Rev. D 86, 014030 (2012) [arXiv:1204.5553 [hep-ph]].

[33] D. L. Yao, M. L. Du, F.-K. Guo and U.-G. Meißner, JHEP 1511, 058 (2015) arXiv:1502.05981 [hep-ph]].

[34] G. Ecker, Phys. Lett. B 336, 508 (1994) hep-ph/9402337].

[35] U.-G. Meißner, G. Müller and S. Steininger, Annals Phys. 279, 1 (2000) hep-ph/9809446.

[36] I. Rosell, P. Ruiz-Femenia and J. Portoles, JHEP 0512, 020 (2005) hep-ph/0510041.

[37] M. Frink, B. Kubis and U.-G. Meißner, Eur. Phys. J. C 25, 259 (2002) hep-ph/0203193.

[38] J. F. Donoghue, E. Golowich and B. R. Holstein, Dynamics of the Standard Model, Cambridge University Press (1992).

[39] G. 't Hooft, Nucl. Phys. B 62, 444 (1973).

[40] J. Bijnens and J. Lu, JHEP 0911, 116 (2009) [arXiv:0910.5424 [hep-ph]]. 